\title{
Natural polyphenol assisted delivery of single-strand oligonucleotides by cationic polymers
}

\author{
Wanwan Shen ${ }^{1} \cdot$ Ruojun Wang ${ }^{1} \cdot$ Qianqian Fan $^{1} \cdot$ Yiwen $\mathrm{Li}^{2} \cdot$ Yiyun Cheng ${ }^{1,3}$
}

Received: 16 January 2020 / Revised: 24 March 2020 / Accepted: 8 April 2020 / Published online: 4 May 2020

(c) The Author(s) 2020. This article is published with open access

\begin{abstract}
Single-strand oligonucleotides provide promising potential as new therapeutics towards various diseases. However, the efficient delivery of oligonucleotide therapeutics is still challenging due to their susceptibility to nuclease degradation and the lack of effective carriers for condensation. In this study, we reported the use of natural polyphenol to facilitate the condensation of single-strand oligonucleotides by cationic polymers. Green tea catechin complexed with single-strand oligonucleotides to form anionic nanoparticles, which were further coated by low molecular weight cationic polymers to increase their cell internalization. The resulting core-shell structured nanoparticles, so-called green nanoparticles (GNPs), showed improved cargo stability, and achieved high efficiency in the delivery of several types of single-strand oligonucleotides including antisense oligonucleotides, anti-miRNA, and DNAzyme. This study provides a facile strategy for the efficient delivery of single-strand oligonucleotides.
\end{abstract}

\section{Introduction}

Oligonucleotide therapeutics including antisense oligonucleotides (ASOs), splice-switching oligonucleotides, steric blockers, aptamers, small Interfering RNA (siRNA), microRNA (miRNA), and other subtypes have shown enormous potential in the treatment of various diseases [1-3]. Since the first ASO was reported to inhibit the expression of sarcoma virus mRNA, oligonucleotide therapeutics have achieved

Supplementary information The online version of this article (https:// doi.org/10.1038/s41434-020-0151-y) contains supplementary material, which is available to authorized users.

Yiwen $\mathrm{Li}$

ywli@scu.edu.cn

$\triangle$ Yiyun Cheng

yycheng@mail.ustc.edu.cn

1 Shanghai Key Laboratory of Regulatory Biology, East China Normal University, Shanghai 200241, China

2 College of Polymer Science and Engineering, State Key Laboratory of Polymer Materials Engineering, Sichuan University, Chengdu 610065, China

3 South China Advanced Institute for Soft Matter Science and Technology, School of Molecular Science and Engineering, South China University of Technology, Guangzhou 510640, China great promise in clinical applications $[4,5]$. Up to now, seven types of oligonucleotide drugs have been approved by FDA, namely fomivirsen (antisense nucleotide), pegaptanib (nucleic acid aptamer), mipomersen (antisense nucleotide), eteplirsen (antisense nucleotides), defibrotide (deoxyribonucleic acid derivatives), nusinersen (antisense nucleotides), and patisirna (siRNA). Note that four of them belong to single-strand oligonucleotides. Besides these drugs, there are still a large number of oligonucleotide drug candidates being widely evaluated in clinical studies $[6,7]$. The common pharmacological challenges for single-strand oligonucleotides are their susceptibility to nuclease degradation, and massive dose requirement [1,8]. During the past years, chemical modification and structural optimization on single-strand oligonucleotides have been proposed to overcome these limitations, i.e., the phosphate backbone of oligonucleotides was replaced by phosphorothioate, phosphodiamine morpholino, and peptide backbones [9-11]. These chemical strategies can alter the charge density and hydrophobicity of single-strand oligonucleotides, and thus improve their nuclease stability and base pairing efficiency.

However, chemically modified single-strand oligonucleotides usually possess relatively low membrane permeability, and limited stability during the long-term therapy [12]. To address those issues, the single-strand oligonucleotides were either conjugated with functional ligands, polymers, and nanoparticles [13,14], or complexed with cationic polymers, 
liposomes, and nanomaterials [15] to increase their cell internalization, stability, and transfection efficiency. Cationic polymers with various chemical structures have widely used for intracellular delivery of biomolecules such as genes and proteins [16-21]. However, these materials have been usually puzzled by their unsatisfied correlations between transfection efficiency and cytotoxicity [22-24].

To break down the transfection efficiency-cytotoxicity correlation of polymers in gene delivery, we reported a facile and efficient siRNA delivery strategy using natural polyphenols and low molecular weight cationic polymers [25]. Natural polyphenols have strong binding affinity with various biomacromolecules such as proteins and nucleic acids via non-covalent interactions [26]. These molecules with potent antioxidant, antibacterial, and antitumor activities were widely used as synthons and function subunits to construct new functional materials for drug delivery [27-37]. One of the natural polyphenol (-)-epigallocatechin gallate (EGCG) was complexed with siRNA to form negatively charged nanoparticles, followed by surface coating on the nanoparticles with a shell of low molecular weight polymers such as $\varepsilon$-poly-L-lysine (PLL, from Streptomyces albus). EGCG protects the bound siRNA from nuclease degradation, and thus improves its stability during intracellular delivery, and the low molecular weight polymers on the particle surface enable efficient internalization but limited cytotoxicity. As a result, the prepared nanoparticles showed excellent genesilencing efficiency both in vitro and in vivo. Considering that EGCG is the major component of green tea, and the nanoparticles are prepared by physical fabrication of several nontoxic compounds, this type of nanoparticles were termed green nanoparticles (GNPs). Since poor stability is the major challenge for single-strand oligonucleotides in gene therapy and most oligonucleotide drugs possess similar physicochemical properties, we hypothesized that this GNPs strategy may be also applicable for the delivery of single-strand oligonucleotides such as ASO, miRNAs, and DNAzymes (Fig. 1).

\section{Materials and methods}

\section{Materials}

EGCG, rhodamine B isothiocyanate (Rho), and 3-(4,5dimethylthiazol-2-yl)-2,5-diphenyltetrazolium bromide (MTT) were obtained from Sigma-Aldrich (St. Louis, MO). PLL (4224 Da) was purchased from Macklin (Shanghai, China). Rabbit monoclonal antibodies against Bcl-2 (catalog number: ab182858) and $\beta$-actin (catalog number: ab16039) were purchased from Abcam, Inc. (Cambridge,

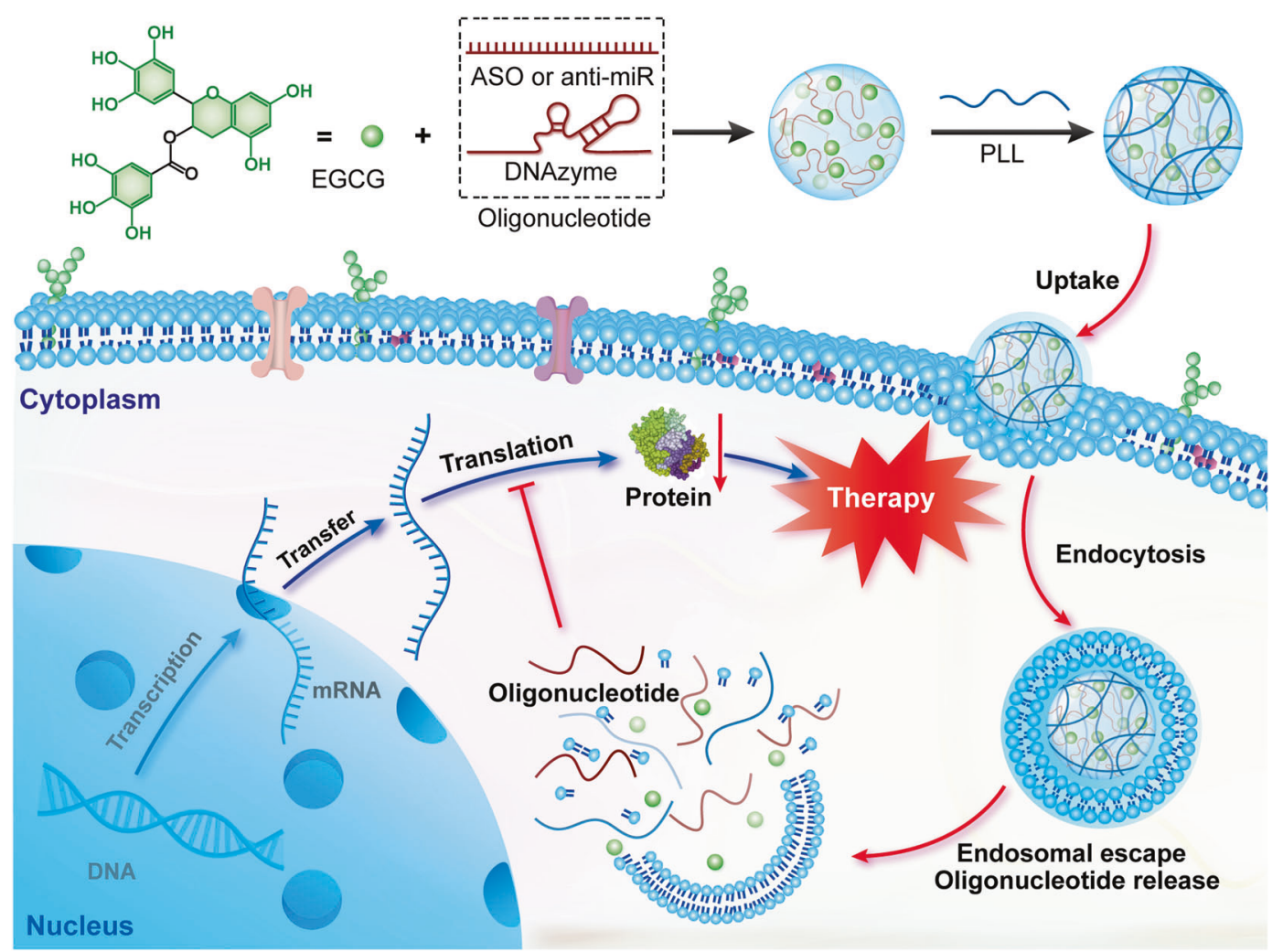

Fig. 1 GNPs mediated gene delivery. Schematic illustration of GNPs for the delivery of single-strand oligonucleotides including ASO, antimiRNA, and DNAzyme. 
UK). Trizol was obtained from NCM Biotech. (Suzhou, China) and Master-mix with SYBR-green kit was obtained from Takara, Inc. (Japan). Commercial transfection reagent Lipofectamine 2000 (LPF) was purchased from Thermo Fisher Scientific, Inc (Shanghai, China) and TransExcellentsiRNA (TE) was purchased from Cenji Biotech. (Shanghai, China). RNase and RNase Inhibitor were obtained from Yeasen Biotech. (Shanghai, China). GelRed was obtained from Beyotime Biotech. (Shanghai, China).

ASO, Ps-ASO, and siRNA specifically targeting firefly luciferase, glyceraldehyde-3-phosphate dehydrogenase (GAPDH), and prolyl hydroxylase-2 (PHD2), Bcl-2 DNAzyme, anti-microRNA-155 (anti-miR-155), scrambled ASO nonspecific to any gene (Sc-ASO), and ASO labeled with carboxyfluorescein at the $5^{\prime}$ end (ASO-FAM) were synthesized by Gene Pharma (Shanghai, China). The sequences of synthesized oligonucleotides were listed in Supplementary Table S1. All the chemicals were used as received without further purification.

\section{Characterization of EGCG/ASO complexes}

Overall, $0.5 \mu \mathrm{g}$ of ASO $(40 \mu \mathrm{M}, 1.88 \mu \mathrm{L})$ was mixed with $5 \mu \mathrm{g}$ of EGCG $(4.36 \mathrm{mM}, 2.5 \mu \mathrm{L})$ in $100 \mu \mathrm{L}$ of diethyl pyrocarbonate-treated water for $20 \mathrm{~min}$ to form EGCG/ASO complexes. Then the sample was diluted to a total volume of $1 \mathrm{~mL}$ by water. The mean hydrodynamic size and zeta potential of yielded nanoparticles were determined by dynamic light scattering (DLS, Zetasizer Nano ZS90, Malvern). The morphology of the formed complexes was observed by transmission electron microscopy (TEM, HT7700, HITACHI, Japan).

\section{Preparation and characterization of GNPs containing ASO}

EGCG/ASO complex containing $0.5 \mu \mathrm{g}$ ASO was prepared as described above, followed by the addition of $5 \mu \mathrm{g}$ PLL $(0.24 \mathrm{mM}, 5 \mu \mathrm{L})$. The mixture was incubated in $100 \mu \mathrm{L}$ of water for $30 \mathrm{~min}$ to form the GNPs. The samples were further diluted to $1 \mathrm{~mL}$ with water. The formed GNPs were characterized by DLS and TEM as described above.

\section{RNase degradation assay}

GNPs containing $0.5 \mu \mathrm{g}$ ASO were prepared as described above. The dose of ASO is $0.5 \mu \mathrm{g}(40 \mu \mathrm{M}, 1.88 \mu \mathrm{L})$, the weight ratio of EGCG $(10.91 \mathrm{mM})$ to ASO is $10: 1$, and the weight ratio of PLL $(1.18 \mathrm{mM})$ to EGCG is fixed at $1: 1$. The volume of mixture in each tube was replenished to $10 \mu \mathrm{L}$ with water. The prepared GNPs were equilibrated for $30 \mathrm{~min}$ and the GNPs were treated with $10 \mu \mathrm{g} / \mathrm{mL}$ RNase $(1 \mathrm{mg} / \mathrm{mL}, 0.1 \mu \mathrm{L})$ for $20 \mathrm{~min}$, and then the activity of
RNase was blocked by $0.1 \mu \mathrm{L}$ RNase inhibitor $(40 \mathrm{U} / \mu \mathrm{L})$. Overall, $11 \mu \mathrm{L}$ mixtures were further run on an agarose gel at $90 \mathrm{~V}(1.5 \% \mathrm{w} / \mathrm{v}$ gel, $10 \mathrm{~min})$ after the addition of $1 \mu \mathrm{L}$ $(100 \mathrm{mg} / \mathrm{mL})$ sodium heparin. The gel was stained by GelRed and visualized by a UV illuminator and the bands were photographed using an UVIpro Gel documentation system. Gray scale calculation was performed using Image J software. Commercial reagents LPF and TE were tested as controls, and the doses were both $2 \mu \mathrm{L}$.

\section{Cell culture and gene-silencing experiments}

HeLa, HeLa-Luc (HeLa cells stably expressing firefly luciferase), and A549 cells were cultured in DMEM containing $10 \%$ fetal bovine serum (FBS), $100 \mathrm{U} \mathrm{mL}^{-1}$ penicillin, and $100 \mathrm{mg} \mathrm{mL}^{-1}$ streptomycin at $37^{\circ} \mathrm{C}$ and $5 \% \mathrm{CO}_{2}$.

The cells were seeded in 24-well plates at a density of $10^{4}$ cells per well and cultured for $24 \mathrm{~h}$ before genesilencing experiments (50\% confluence). Overall, $1.88 \mu \mathrm{L}$ oligonucleotides $(40 \mu \mathrm{M}, 0.5 \mu \mathrm{g}$ ASO-Luci for HeLa-Luc cells, $0.5 \mu \mathrm{g}$ ASO-GAPDH and ASO-PHD2 for HeLa cells, $0.8 \mu \mathrm{g}$ DNAzyme-Bcl-2 for HeLa cells, and $0.5 \mu \mathrm{g}$ antimiR-155 for A549 cells) were mixed with freshly prepared $2.5 \mu \mathrm{L}$ EGCG $(4.36 \mathrm{mM}$ ) for $20 \mathrm{~min}$ (the EGCG/oligonucleotide weight ratio of 10:1 for ASO and anti-miR-155, and 6.25:1 for DNAzyme-Bcl-2), followed by incubation with PLL (0.24 mM, PLL/EGCG weight ratio of $1: 1)$ to yield GNPs, and further diluted with $100 \mu \mathrm{L}$ FBS-free media (10 mM HEPES buffer was added to maintain the medium $\mathrm{pH}$ at 7.4). The GNPs solutions were incubated at the room temperature for $30 \mathrm{~min}$, and further added with $150 \mu \mathrm{L}$ culture media before added with cells. The cells were cultured with GNPs for $6 \mathrm{~h}$. After that, $500 \mu \mathrm{L}$ media containing 10\% FBS were added into the wells and the gene-silencing experiments were continued for $18 \mathrm{~h}$. The final concentration of oligonucleotides in the culture medium was $100 \mathrm{nM}$. Three repeats were conducted for each sample in three independent experiments.

The luciferase activity in HeLa-Luc cells was measured as previous study [25]. The expression levels of GAPDH, PHD2, and Bcl-2 mRNA in the treated cells were characterized by real-time reverse transcription quantitative PCR (RT-qPCR). Overall, $0.5 \mu \mathrm{g}$ extracted RNA in the treated cells lysis was reverse-transcribed into cDNA and quantitative analyzed by qPCR (QuantStudio 3 Real-Time PCR Systems, Thermo Fisher Scientific) with the specific primers and SYBR-green kit. The related primers for each target gene were shown in Supplementary Table S2.

The Bcl-2 protein levels in the treated cells was analyzed by western blot. Generally, $50 \mu \mathrm{g}$ total protein per lane were separated on $12 \%$ SDS-PAGE gels and transferred onto a PVDF membrane. The membrane was incubated with rabbit monoclonal antibodies against Bcl-2 overnight at $4{ }^{\circ} \mathrm{C}$, and 
further incubated with IRDye 800 donkey anti-rabbit (LICOR, USA) for $1 \mathrm{~h}$. The protein bands on the gel were visualized using an Odyssey CLx infrared imaging system (LI-COR, USA). $\beta$-actin was used as the loading control.

Cell apoptosis in the treated cells were measured by an annexin V-FITC/propidium iodide (PI) apoptosis detection kit. The treated cells were stained by FITC-labeled Annexin $\mathrm{V}$ and PI for $15 \mathrm{~min}$ at the room temperature in the dark. The samples were quantitatively analyzed by flow cytometry (BD FACS Calibur, San Jose).

\section{Statistical analysis}

Adequate sample size was determined according to the previous studies $[23,25]$ that performed analogous experiments. Data are represented as the derive average \pm standard deviation throughout the manuscript. The variance was similar between the groups that are being statistically compared. Comparisons of data from tests and controls
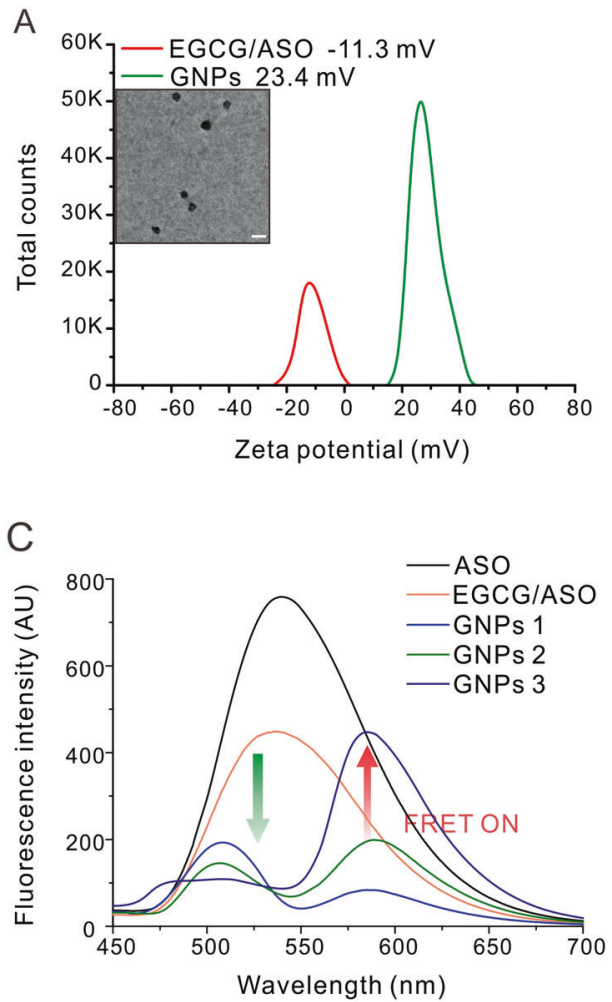

Fig. 2 The characterizations of the prepared GNPs. a Zeta potential of EGCG/ASO complexes and GNPs. TEM image of EGCG/ASO complexes was insert in the picture. Scale bar is $100 \mathrm{~nm}$. b DLS and TEM image of GNPs consisted of PLL, EGCG, and ASO. The dose of ASO was $0.5 \mu \mathrm{g}(133 \mathrm{nM})$, EGCG to ASO weight ratio was $10: 1$, and PLL to EGCG weight ratio was $1: 1$. Scale bar is $100 \mathrm{~nm}$. c Fluorescence spectra of ASO-FAM, EGCG/ASO-FAM, and GNPs consisted of ASO-FAM, EGCG, and PLL-Rho. The dose of ASO-FAM in each well was $1.0 \mu \mathrm{g}(267 \mathrm{nM})$, the weight ratio of EGCG to ASO-FAM was $5: 1,10: 1,20: 1$ for GNPs 1 , GNPs 2, and GNPs 3, respectively. PLL to EGCG weight ratio was 1:1 and EGCG to ASO-FAM weight were analyzed for statistical significance by a one-sided Student's $t$ test using MS Excel. For all, $p<0.05$ was considered statistically significant. $* p<0.05$; $* * p<0.01$; $* * * p<0.001$. In this study, experiments were performed on at least three independent occasions, and no randomization and blinding were used. These tests were chosen since they best match the assumptions of the experiments.

\section{Results and discussion}

We first investigated the formation of EGCG/single-strand oligonucleotide ASO complexes using DLS and TEM. It was found that EGCG and ASO formed negatively charged nanoparticles in aqueous solution $(-11.3 \mathrm{mV}$, Fig. 2a). After coating with PLL, nanoparticles with an average hydrodynamic size of $127 \mathrm{~nm}$ were observed (Fig. 2b), and the zeta potential of particles increased from -11.3 to $23.4 \mathrm{mV}$ (Fig. 2a), suggesting the successful coating of
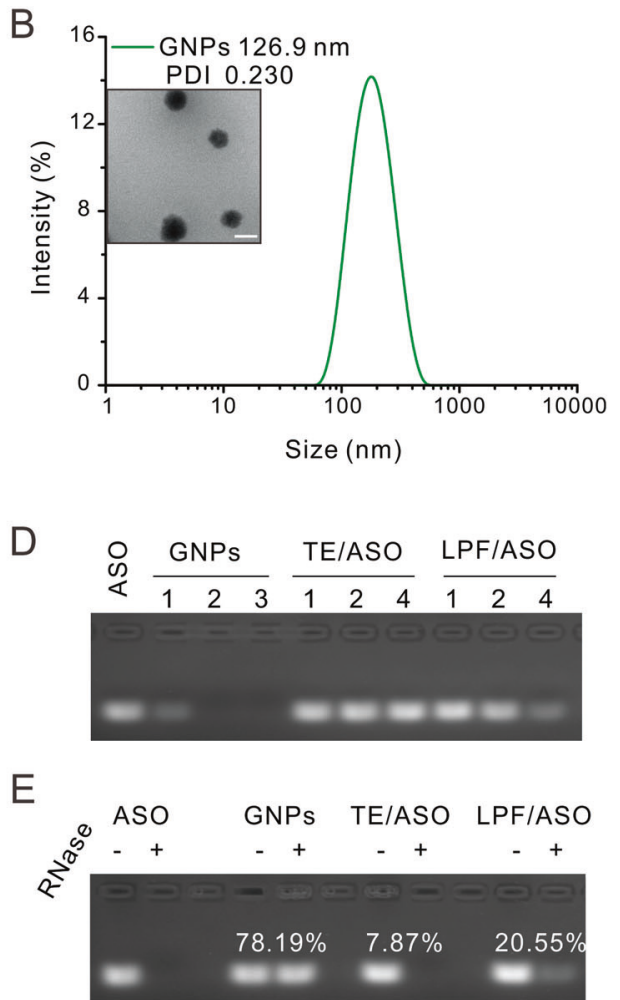

ratio was 20:1 for the EGCG/ASO complex. d Gel electrophoresis of GNPs. The dose of ASO was $0.5 \mu \mathrm{g}$, and the weight ratio of EGCG to ASO was 5:1, 10:1, 20:1 for GNPs 1, GNPs 2, and GNPs 3, respectively. PLL to EGCG weight ratio was 1:1. TE/ASO and LPF/ASO complexes were tested as controls, and the doses of TE and LPF were 1, 2, and $4 \mu \mathrm{L}$. e Gel electrophoresis of RNase-treated GNPs. The numbers on the band represent the percent of retained ASO, which were calculated by ImageJ software. The material doses in GNPs were equal to those in GNPs 2 in (d). The doses of TE and LPF were $2 \mu \mathrm{L}$, and the concentration of RNase was $10 \mu \mathrm{g} / \mathrm{mL}$. 
A
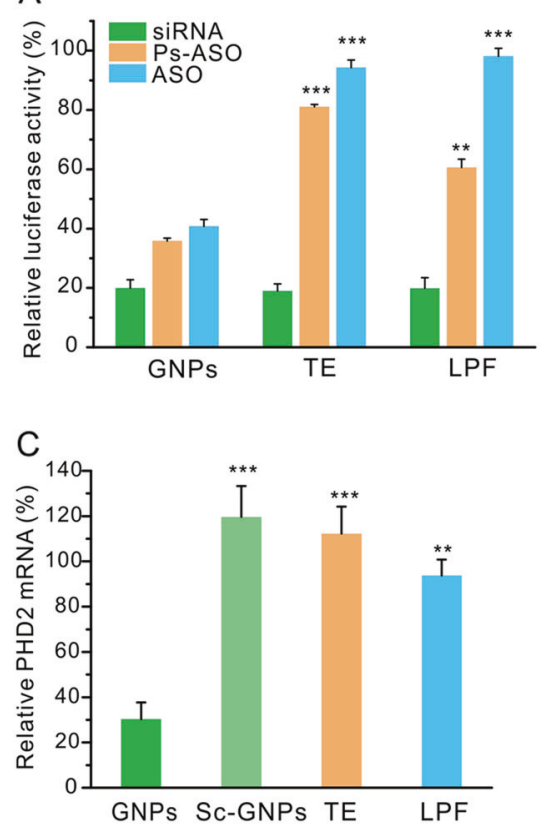

Fig. 3 Efficiency of GNPs in the delivery of oligonucleotides. a Efficiency of GNPs in the delivery of siRNA, ASO, and Ps-ASO into Hela-Luc cells for $24 \mathrm{~h}$. The concentration of oligonucleotides in each well was fixed at $100 \mathrm{nM}$. The dose of EGCG in each well was $5 \mu \mathrm{g}$, and the weight ratio of PLL to EGCG was 1:1. GAPDH (b) and PHD2 (c) knockdown efficiency of GNPs in HeLa cells. GNPs were consisted of ASO, EGCG, and PLL. The concentration of ASO was 100 $\mathrm{nM}(1.0 \mu \mathrm{g})$, the weight ratio of EGCG to ASO was 10:1, and the

PLL on the EGCG/ASO complexes. We further investigated the complexation process by fluorescence resonance energy transfer (FRET) experiment. As shown in Fig. 2c, the fluorescence of carboxyfluorescein labeled ASO (ASO-FAM) was significantly decreasing after the addition of EGCG due to the formation of ASO-FAM complexes. After coating with rhodamine-labeled PLL (PLL-Rho), the fluorescence intensity of ASO-FAM was further decreasing, while that of PLL-Rho was increasing (Fig. 2c). The FRET signals increased with increasing EGCG/PLL to ASO weight ratios, and this result confirmed the formation of PLL-coated EGCG/ASO nanoparticles (GNPs: the PLL/ EGCG/ASO weight ratio is 5:5:1, 10:10:1 and 20:20:1, respectively for GNPs 1-3) in the solutions. Note that the distinct fluorescence spectra of GNPs 1-3 were observed, which might be due to their different particle size and aggregated structures. We also investigated the ASO-binding capability of GNPs by gel electrophoresis. Two commercial gene transfection reagents TE (a polymer-based reagent) and LPF (a lipid-based reagent) were used as the controls. The ASO was efficiently bound in the nanoparticles when the EGCG to ASO weight ratio was above 10:1 (Fig. 2d), while TE and LPF failed to efficiently bind with ASO under the electrophoresis condition. We further investigated the
B

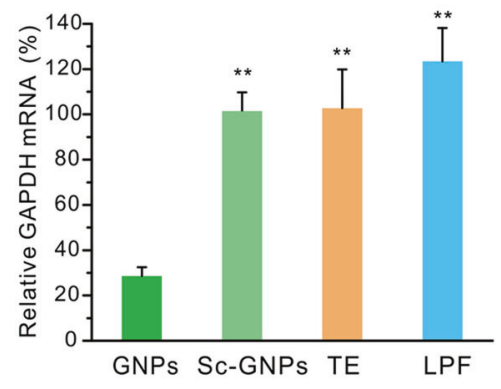

D

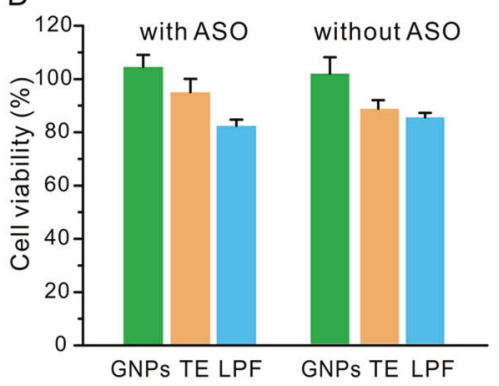

weight ratio of PLL to EGCG was 1:1. Data are representative of three independent experiments performed with three technical duplicates in (a-c). d Cell viability of HeLa cells treated with GNPs and EGCG/ PLL for $24 \mathrm{~h}$. The concentrations of the materials were equal to those in $(\mathbf{b}, \mathbf{c})$. TE and LPF were tested as controls. The doses of TE and LPF in each well were both $2.0 \mu \mathrm{L}$. Data are representative of three independent experiments performed with five technical duplicates. $* * p<0.01, * * * p<0.001$ analyzed by Student's $t$ test.

protective effects of GNPs on ASO via RNase degradation experiment. The remained ASO in the GNPs was 78.19\% after RNase $(10 \mu \mathrm{g} / \mathrm{mL})$ treatment for $20 \mathrm{~min}$, while those of TE and LPF were $7.87 \%$ and $20.55 \%$, respectively (Fig. 2e), which suggested that GNPs can efficiently protect ASO from degradation by RNase and increase the tolerance of ASO to nuclease. The increased stability of ASO in GNPs might be due to the core-shell structure of GNPs, in which the oligonucleotides located in the interior of formed nanoparticles [25]. Since GNPs 2 with the PLL/EGCG/ASO weight ratio of 10:10:1 showed effective ASO binding and complex stability, this formulation was further used in subsequent gene transfection experiments.

We then tested the efficiency of GNPs in the delivery of ASO into HeLa-Luc cells to knockdown the firefly luciferase gene. Three type of oligonucleotides including nonmodified ASO, phosphorothioate backbone ASO (Ps-ASO), and double-strand siRNA targeting luciferase were tested as cargo molecules. The antisense strand of these three oligonucleotides is identical to each other and the concentration of oligonucleotides was fixed at $100 \mathrm{nM}$. As shown in Fig. 3a, GNPs, TE, and LPF exhibited high gene-silencing efficiency in the delivery of siRNA. However, the gene knockdown efficiencies by TE was decreased to $19 \%$ and 
A

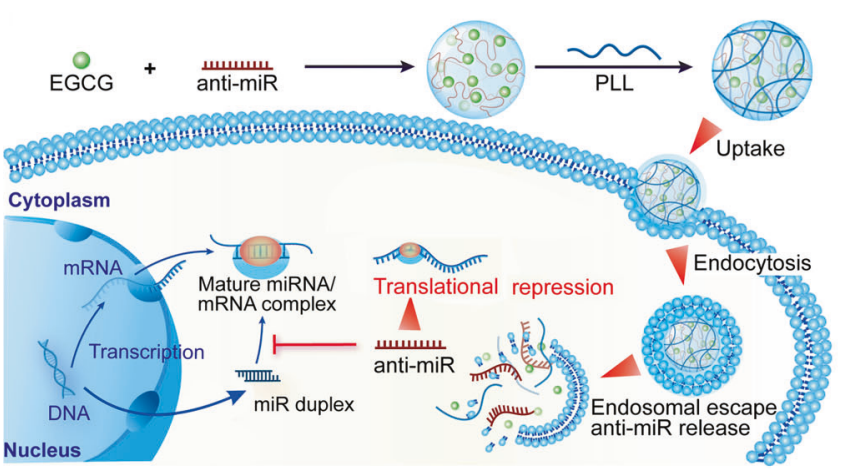

C
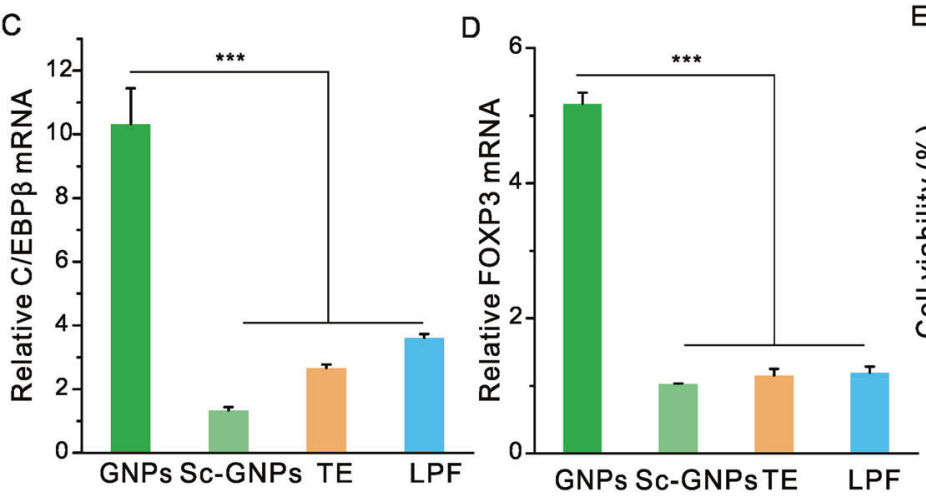
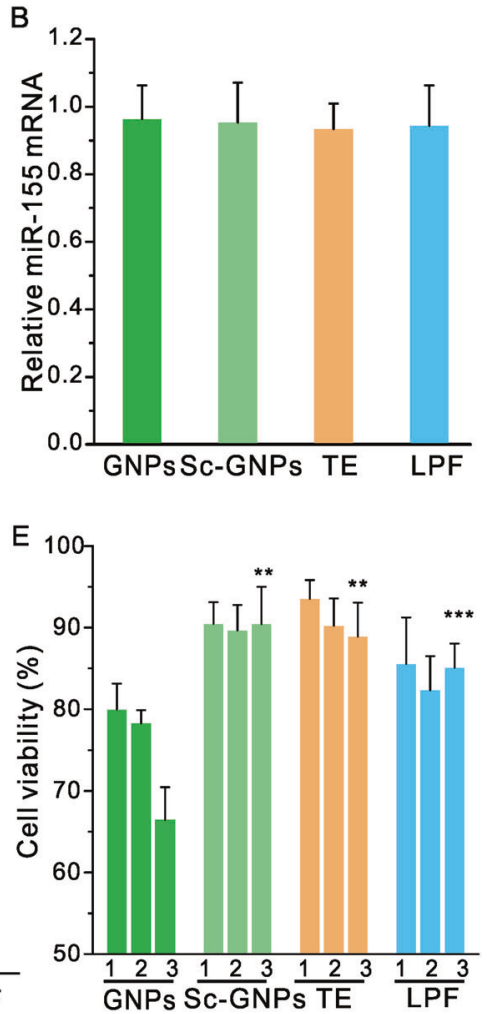

Fig. 4 Efficiency of GNPs in the delivery of anti-miRNA into A549 cells. a Schematic illustration of anti-miRNA delivery by GNPs. The expression levels of miR-155 (b), C/EBP $\beta$ (c) and FOXP3 (d) in GNPs-treated A549 cells for $24 \mathrm{~h}$. The dose of anti-miR-155 was 0.5 $\mu \mathrm{g}$ in each well $(100 \mathrm{nM})$, the weight ratio of EGCG to anti-miR-155 was 10:1, and the weight ratio of PLL to EGCG was $1: 1$. *** $p<0.001$ analyzed by Student's $t$ test. Results represent three independent biological PCR reactions performed in triplicate. in (b-d). e Viability of
GNPs-treated A549 cells for $24 \mathrm{~h}$. The doses of anti-miR-155 in 1, 2, 3 were $0.5 \mu \mathrm{g}$ in each well $(100 \mathrm{nM}), 1.0 \mu \mathrm{g}(200 \mathrm{nM})$, and $1.5 \mu \mathrm{g}(300$ $\mathrm{nM}$ ), respectively. The dose of EGCG was $5.0 \mu \mathrm{g}$, the weight ratio of PLL to EGCG was 1:1, and the doses of TE and LPF were $2.0 \mu \mathrm{L}$. Data are representative of three independent experiments performed with five technical duplicates. $* * p<0.01$, ***p $p 0.001$ between GNPs 3 and other control groups analyzed by Student's $t$ test.

miRNA usually inhibits the translation of target mRNA by complementary pairing, and its function can be inhibited by anti-miRNA (anti-miR), a type of steric blocker ASOs [38-40] (Fig. 4a). MiR-155, a typical multifunctional miRNA, is related to tumorigenesis and metastasis in a variety of cancer cells and has been recognized as a novel tumor biomarker for cancer therapy [41, 42]. Anti-miRNA-155 can be used to inhibit the function of oncogenic miRNA-155. The pre-complementary pairing of anti-miRNA with miRNA hinders the recognition of oncogene miRNA-155 with its target mRNA such as transcription factor CCAAT enhancer binding protein $\beta(\mathrm{C} / \mathrm{EBP} \beta)$ and forkhead transcription factor FOXP3 to prevent it from functioning properly [43]. Herein, GNPs were further used to deliver anti-miR-155 to inhibit the function of miR-155. The mRNA expression level of miRNA-155 was scarcely changed after treatment (Fig. 4b), but the levels of target gene C/EBP $\beta$ and FOXP3 in the cells were increased by tenfold and fivefold, respectively (Fig. 4c, d), which were again significantly higher than those by control materials (TE and LPF). Sc-GNPs containing nonsense anti-miRNA did not cause significant changes in the 
A

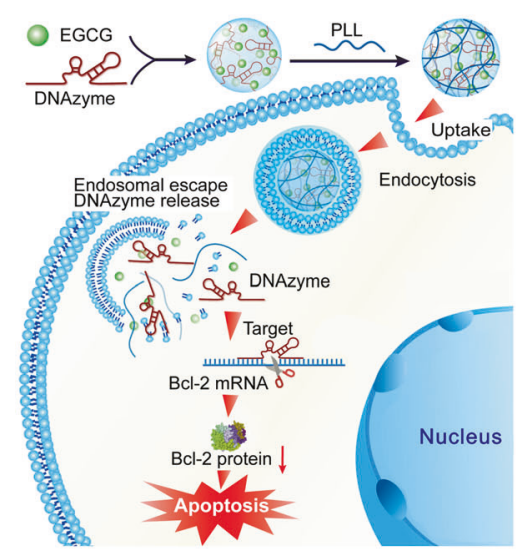

B

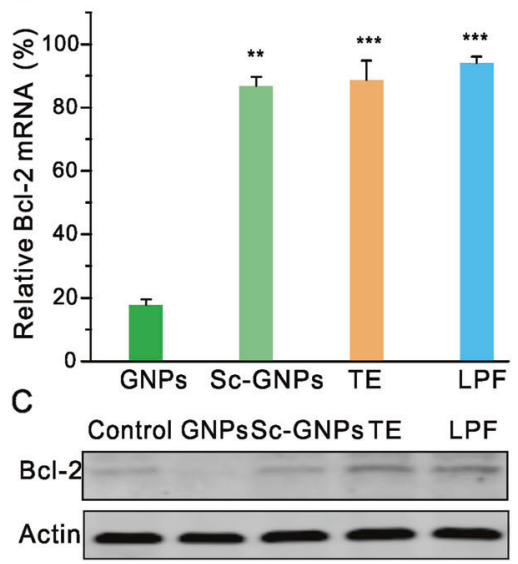

D

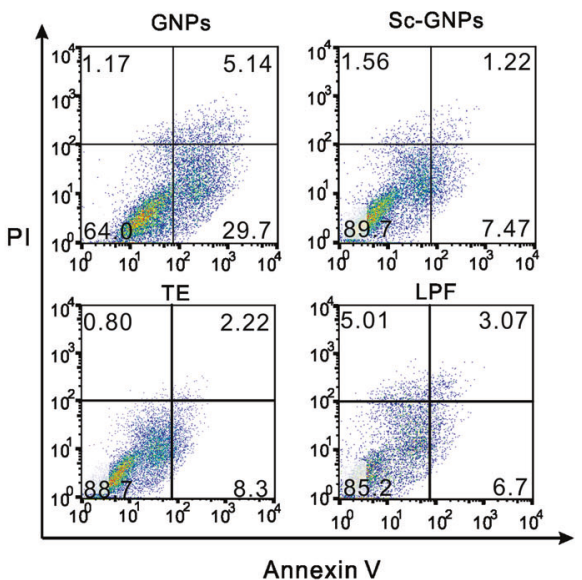

Fig. 5 Efficiency of GNPs in the delivery of DNAzyme into HeLa cells. a Schematic illustration of Bcl-2 DNAzyme delivery by GNPs. b Bcl-2 mRNA expression in GNPs-treated HeLa cells for $24 \mathrm{~h}$. Results represent three independent biological PCR reactions performed in triplicate. $\mathbf{c}$ Representative $\mathrm{Bcl}-2$ protein expressions in the

expressions corresponding target genes. The results suggested that anti-miR-155 was successfully delivered into cells and specifically bound with miR-155 to inhibit its function. As a result, the viability of A549 cells was significantly reduced (Fig. 4e). All the evidences above demonstrated that GNPs can efficiently deliver anti-miRNA into cells to regulate the expressions of downstream genes.

In comparison with ASOs, DNAzyme has lower offtarget effects due to its special internal structure, which consists of both binding sites and catalytic sites within the molecule. Numerous clinical results have pointed out that DNAzyme is well-tolerated in humans [44-46]. Bcl-2 is an oncogene and numerous researches have been focused on the downregulation of $\mathrm{Bcl}-2$ by oligonucleotides to induce apoptosis in cancer cells [47-49]. We finally tested the efficiency of GNPs in the delivery of DNAzyme targeting Bcl-2 on HeLa cells (Fig. 5a). As shown in Fig. 5b, GNPs containing the DNAzyme significantly reduced the expression of Bcl-2 mRNA, with an efficiency higher than $80 \%$. However, GNPs containing nonsense DNAzyme failed to downregulate the target gene, indicating the successful delivery of Bcl-2 DNAzyme into the cells. Furthermore, the GNPs containing Bcl-2 DNAzyme successfully inhibited Bcl-2 protein levels (Fig. 5c) and caused significantly increased apoptosis in the cells (Fig. 5d).

\section{Conclusions}

Inspired by the high efficiency of GNPs in siRNA delivery, we investigated the behaviors of GNPs in the delivery of several single-strand oligonucleotides including ASO, antimicroRNA, and DNAzyme. The results revealed that GNPs treated cells. d Flow cytometry analysis of treated HeLa cells stained by annexin $\mathrm{V}$ and propidium iodide. The concentration of DNAzyme in each well fixed at $100 \mathrm{nM}(0.8 \mu \mathrm{g})$, the dose of EGCG and PLL in each well was both $5.0 \mu \mathrm{g}$. The doses of TE and LPF were $2.0 \mu \mathrm{L}$. $* * p<0.01, * * * p<0.001$ analyzed by Student's $t$ test.

can efficiently protect the single-strand nucleic acids from enzymatic degradation, and successfully delivered these oligonucleotides into cells to exert their biofunctions. This study further confirmed that GNPs could be developed as a facile, robust, and efficient strategy in the delivery of oligonucleotide therapeutics. Our future work will test the efficiency of these GNPs in the delivery of single-strand oligonucleotides in vivo and evaluate their potentials in clinical translation.

\section{Data availability}

All relevant data generated in this study are included in the published article and supplementary information.

Funding This study was supported from the National Key R\&D Program of China, Synthetic Biology Research (No. 2019YFA0904500), the National Natural Science Foundation of China (21725402 and 21774079), the Shanghai Municipal Science and Technology Commission (17XD1401600 and 188014580), and the Guangdong Innovative and Entrepreneurial Research Team Program (2016ZT06C322).

Author contributions WS carried out the preparation, characterization, and biological evaluation of GNPs. RW and QF performed part of the synthesis and characterization study. YL and YC designed and supervised the study, and wrote the manuscript.

\section{Compliance with ethical standards}

Conflict of interest The authors declare that they have no conflict of interest.

Publisher's note Springer Nature remains neutral with regard to jurisdictional claims in published maps and institutional affiliations. 
Open Access This article is licensed under a Creative Commons Attribution 4.0 International License, which permits use, sharing, adaptation, distribution and reproduction in any medium or format, as long as you give appropriate credit to the original author(s) and the source, provide a link to the Creative Commons license, and indicate if changes were made. The images or other third party material in this article are included in the article's Creative Commons license, unless indicated otherwise in a credit line to the material. If material is not included in the article's Creative Commons license and your intended use is not permitted by statutory regulation or exceeds the permitted use, you will need to obtain permission directly from the copyright holder. To view a copy of this license, visit http://creativecommons.org/licenses/by/4.0/.

\section{References}

1. Khvorova A, Watts JK. The chemical evolution of oligonucleotide therapies of clinical utility. Nat Biotechnol. 2017;35:238-48.

2. Benizri S, Gissot A, Martin A, Vialet B, Grinstaff MW, Barthélémy P. Bioconjugated oligonucleotides: recent developments and therapeutic applications. Bioconjug Chem. 2019;30:366-83.

3. Crooke ST. Molecular mechanisms of antisense oligonucleotides. Nucleic Acid Ther. 2017;27:70-7.

4. Agrawal S. Remembering Paul C. Zamecnik, M.D., 'father of antisense' (1912-2009). Oligonucleotides. 2010;20:47-50.

5. Agrawal S, Zamecnik PC. History of antisense oligonucleotides. Antisense Ther. 2003;1:1-12.

6. Corey DR. Nusinersen, an antisense oligonucleotide drug for spinal muscular atrophy. Nat Neurosci. 2017;20:497-9.

7. Bennett CF, Baker BF, Pham N, Swayze E, Geary RS. Pharmacology of antisense drugs. Annu Rev Pharmacol Toxicol. 2017;57:81-105.

8. Sun Y, Zhao Y, Zhao X, Lee RJ, Teng L, Zhou C. Enhancing the therapeutic delivery of oligonucleotides by chemical modification and nanoparticle encapsulation. Molecules. 2017;22:1724.

9. Hassler MR, Turanov AA, Alterman JF, Haraszti RA, Coles AH, Osborn MF, et al. Comparison of partially and fully chemicallymodified siRNA in conjugate-mediated delivery in vivo. Nucleic Acids Res. 2018;46:2185-96.

10. Flierl U, Nero TL, Lim B, Arthur JF, Yao Y, Jung SM, et al. Phosphorothioate backbone modifications of nucleotide-based drugs are potent platelet activators. J Exp Med. 2015;212:129-37.

11. Zhou H, Muntoni F. Morpholino-mediated exon inclusion for SMA. Methods Mol Biol. 2018;1828:467-77.

12. Craig K, Abrams M, Amiji M. Recent preclinical and clinical advances in oligonucleotide conjugates. Expert Opin Drug Del. 2018;15:629-40.

13. Husser C, Brink A, Zell M, Müller MB, Koller E, Schadt S. Identification of GalNAc-conjugated antisense oligonucleotide metabolites using an untargeted and generic approach based on high resolution mass spectrometry. Anal Chem. 2017;89:6821-6.

14. Nakagawa O, Ming X, Carver K, Juliano R. Conjugation with receptor-targeted histidine-rich peptides enhances the pharmacological effectiveness of antisense oligonucleotides. Bioconjug Chem. 2014;25:165-70.

15. Juliano RL. The delivery of therapeutic oligonucleotides. Nucleic Acids Res. 2016;44:6518-48.

16. Yang J, Zhang Q, Chang H, Cheng Y. Surface-engineered dendrimers in gene delivery. Chem Rev. 2015;115:5274-5300.

17. Wang M, Liu H, Li L, Cheng Y. A fluorinated dendrimer achieves excellent gene transfection efficacy at extremely low nitrogen to phosphorus ratios. Nat Commun. 2014;5:3053.

18. Lv J, Fan Q, Wang H, Cheng Y. Polymers for cytosolic protein delivery. Biomaterials. 2019;218:119358.

19. Liu C, Wan T, Wang H, Zhang S, Ping Y, Cheng Y. A boronic acid-rich dendrimer with robust and unprecedented efficiency for cytosolic protein delivery and CRISPR-Cas9 gene editing. Sci Adv. 2019;5:eaaw8922.

20. Zhang Z, Shen W, Ling J, Yan Y, Hu J, Cheng Y. The fluorination effect of fluoroamphiphiles in cytosolic protein delivery. Nat Commun. 2018;9:1377.

21. Cheng Y, Ren L, Lv J, Wang H. A coordinative dendrimer achieves excellent efficiency in cytosolic protein and peptide delivery. Angew Chem Int Ed. 2020;59:4711-19.

22. Wang H, Chang H, Zhang Q, Cheng Y. Fabrication of lowgeneration dendrimers into nanostructures for efficient and nontoxic gene delivery. Top Curr Chem. 2017;375:62.

23. Wang $\mathrm{H}$, Wang $\mathrm{Y}$, Wang $\mathrm{Y}, \mathrm{Hu} \mathrm{J}, \mathrm{Li} \mathrm{T}$, Liu $\mathrm{H}$, et al. Self-assembled fluorodendrimers combine the features of lipid and polymeric vectors in gene delivery. Angew Chem Int Ed. 2015;54:11647-51.

24. Liu H, Wang H, Yang W, Cheng Y. Disulfide cross-linked low generation dendrimers with high gene transfection efficacy, low cytotoxicity, and low cost. J Am Chem Soc. 2012;134: 17680-7.

25. Shen W, Wang Q, Shen Y, Gao X, Li L, Yan Y, et al. Green tea catechin dramatically promotes RNAi mediated by low-molecularweight polymers. ACS Cent Sci. 2018;4:1326-33.

26. Liu C, Shen W, Li B, Li T, Chang H, Cheng Y. Natural polyphenols augment cytosolic protein delivery by a functional polymer. Chem Mater. 2019;31:1956-65.

27. Wang C, Sang H, Wang Y, Zhu F, Hu X, Wang X, et al. Foe to friend: supramolecular nanomedicines consisting of natural polyphenols and bortezomib. Nano Lett. 2018;18: $7045-51$

28. Li M, Wang $\mathrm{H}, \mathrm{Hu}$ J, Hu J, Zhang S, Yang Z, et al. Smart hydrogels with antibacterial properties built from all natural building blocks. Chem Mater. 2019;31:7678-85.

29. Cheng X, Li M, Wang H, Cheng Y. All-small-molecule dynamic covalent gels with antibacterial activity by boronate-tannic acid gelation. Chin Chem Lett. 2020;31:869-74.

30. Zhou Z, Yan Y, Wang L, Zhang Q, Cheng Y. Melanin-like nanoparticles decorated with an autophagy-inducing peptide for efficient targeted photothermal therapy. Biomaterials. 2019;203: $63-72$.

31. Wang X, Wang C, Wang X, Wang Y, Zhang Q, Cheng Y. A polydopamine nanoparticle-knotted poly(ethylene glycol) hydrogel for on-demand drug delivery and chemo-photothermal therapy. Chem Mater. 2017;29:1370-6.

32. Wang C, Wang D, Dai T, Xu P, Wu P, Zou Y, et al. Skin pigmentation-inspired polydopamine sunscreens. Adv Funct Mater. 2018;28:1802127.

33. Dai Q, Geng H, Yu Q, Hao J, Cui J. Polyphenol-based particles for theranostics. Theranostics. 2019;9:3170-90.

34. Xu LQ, Neoh KG, Kang ET. Natural polyphenols as versatile platforms for material engineering and surface functionalization. Prog Polym Sci. 2018;87:165-96.

35. Faure E, Falentin-Daudré C, Jérôme C, Lyskawa J, Fournier D, Woisel $\mathrm{P}$, et al. Catechols as versatile platforms in polymer chemistry. Prog Polym Sci. 2013;38:236-70.

36. Xiang S, Yang $P$, Guo $H$, Zhang $S$, Zhang $X$, Zhu F, et al. Green tea makes polyphenol nanoparticles with radical-scavenging activities. Macromol Rapid Commun. 2017;38:1700446.

37. Ye Q, Zhou F, Liu W. Bioinspired catecholic chemistry for surface modification. Chem Soc Rev. 2011;40:4244-58.

38. Rupaimoole R, Slack FJ. MicroRNA therapeutics: towards a new era for the management of cancer and other diseases. Nat Rev Drug Discov. 2017;16:203-21.

39. Ambros V. The functions of animal microRNAs. Nature. 2004; 431:350-5. 
40. Cheng CJ, Bahal R, Babar IA, Pincus Z, Barrera F, Liu C, et al. MicroRNA silencing for cancer therapy targeted to the tumor microenvironment. Nature. 2015;518:107-10.

41. Elton TS, Selemon H, Elton SM, Parinandi NL. Regulation of the miR-155 host gene in physiological and pathological processes. Gene. 2013;532:1-12.

42. Xiaoyan W, Pais EMA, Lan L, Jingrui C, Lin M, Fordjour PA, et al. MicroRNA-155: a novel armamentarium against inflammatory diseases. Inflammation. 2017;40:708-16.

43. Weidhaas JB, Liang X, Saltzman WM, Booth CJ, Slack FJ, Cheng CJ, et al. Nanoparticle-based therapy in an in vivo microRNA-155 (miR-155)-dependent mouse model of lymphoma. Proc Natl Acad Sci USA. 2012;109:E1695-E704.

44. Liu M, Chang D, Li Y. Discovery and biosensing applications of diverse RNA-cleaving DNAzymes. Acc Chem Res. 2017;50:2273-83.
45. Liu H, Yu X, Chen Y, Zhang J, Wu B, Zheng L, et al. Crystal structure of an RNA-cleaving DNAzyme. Nat Commun. 2017; 8:2006.

46. Zhou W, Ding J, Liu J. Theranostic DNAzymes. Theranostics. 2017;7:1010-25.

47. Yang X, Li Z, Zhang L, He J, Sun L-Q. Selection and antitumor activity of anti-Bcl-2 DNAzymes. Biochem Biophys Res Commun. 2016;479:544-50.

48. Shen W, Wang H, Ling-Hu Y, Lv J, Chang H, Cheng Y. Screening of efficient polymers for siRNA delivery in a library of hydrophobically modified polyethyleneimines. J Mater Chem B. 2016;4:6468-74.

49. Delbridge ARD, Grabow S, Strasser A, Vaux DL. Thirty years of BCL-2: Translating cell death discoveries into novel cancer therapies. Nat Rev Cancer. 2016;16:99-109. 